\title{
Effects of body weight and level of deprivation on activity of rats with lesions of the ventromedial hypothalamus
}

\author{
RICHARD YOUNG and JOSEPH H. PORTER \\ Virginia Commonwealth University, Richmond, Virginia 23284
}

\begin{abstract}
Nine VMH-lesioned rats were tested for photocell and open-field activity at nonobese and obese body weights at $0 \%, 10 \%$, and $20 \%$ deprivation. An increased activity level was displayed by the nonobese VMH animals, but only under food-deprivation conditions. Conversely, a decreased activity level was displayed by obese VMH animals, but once again, only during deprived testing conditions. The results are discussed in terms of recent findings regarding the VMH-lesioned rat's activity level and willingness to work for food.
\end{abstract}

The term "ventromedial hypothalamus" (VMH) is used to describe that portion of the hypothalamus which, when lesioned, produces hyperphagia. The hyperphagia is accompanied by a very rapid weight gain, often resulting in a doubling of the animal's original body weight (Brobeck, Tepperman, \& Long, 1943; Hetherington \& Ranson, 1940). The VMH includes, but is not limited to, the VM nuclei. This distinction is important, since recent work (Gold, 1973) has questioned the role of the VM nuclei in hypothalamic hyperphagia.

One might predict that an animal which overeats (as a result of VMH lesions) would also demonstrate an increased motivation on food-directed (barpressing) tasks, but this has yet to be convincingly demonstrated. It has been shown that rats with VMH lesions display a decrease in motivation (e.g., Miller, Bailey, \& Stevenson, 1950; Teitelbaum, 1957), an increase in motivation (e.g., Falk, 1961; Kent \& Peters, 1973), or no change in motivation (e.g., Porter \& Allen, 1972; Sclafani, 1971). Several variables have been mentioned by various investigators (e.g., Marks \& Remley, 1972; Porter \& Allen, 1977; Sclafani, 1976; Singh, 1970) to clarify these discrepant results. These factors include the body weight of the VMHlesioned rat at the time of testing, the amount of preoperative or postoperative training, and the level of deprivation at the time of testing. Marks and Remley (1972), Porter, Allen, and Arazie (1974), and Wampler (1973) have reported that at nonobese weight levels the performance of VMH-lesioned rats is similar to or higher than that of normal rats, but at obese weight levels they typi-

This study was based upon a thesis submitted by the senior author in partial fulfillment of the requirments for the Master of Science degree in Psychology at Virginia Commonwealth University. Reprint requests should be sent to Joseph H. Porter, Department of Psychology, Virginia Commonwealth University, Richmond, Virginia 23284. cally exhibit a performance decrement, although Beatty (1973), Porter and Allen (1977), and Sclafani and Kluge (1974) have reported exceptions. King and Gaston (1973) and Singh (1973) showed that VMHlesioned rats with preoperative training responded at control levels on fixed ratio (FR) schedules of reinforcement. When no preoperative training is given, a performance decrement on FR schedules has typically been found (Hamilton, 1963; Singh, 1970; Storlien \& Albert, 1972; Teitelbaum, 1957). Finally, the level of deprivation at the time of testing has been implicated as a variable of importance. Under deprived conditions, VMH-lesioned and control rats had similar levels of performance, but under nondeprived conditions the VMH-lesioned rats demonstrated a higher level of performance than did the control rats (Kent \& Peters, 1973; Panksepp \& Dickenson, 1972; Sclafani \& Kluge, 1974). Recently, Porter and Allen (1977) have investigated these variables in a factorial design and found that, under deprived and nondeprived testing conditions, nonobese VMH rats did not respond differently from control rats on a VI 1-min food reinforcement schedule. However, a performance decrement was found with obese VMH rats, but only under deprived testing conditions. Porter and Allen concluded that "VMH lesions per se, do not produce a decrease in food motivated performance, but that the performance deficits are a function of the body weight of the animal and the level of deprivation at the time of testing"' (p. 238).

Ventromedial hypothalamic lesions also have been shown to affect spontaneous activity, as measured by stabilimeter or photocell activity cages, open-field devices, and by running wheels. However, whether or not these lesions consistently produce a significant effect on total activity is still open to experimental exploration. For example, decreased activity of VMHlesioned animals has been reported in stabilimetertype living cages (Teitelbaum, 1957), photocell activ- 
ity cages (Storlien \& Albert, 1972), and in running wheels or revolving drums (Brooks, 1946; Hetherington \& Ranson, 1942; Sclafani, 1971). Conversely, increased activity in the open field (Green, 1967; Grossman, 1972) as well as no change in activity (Sclafani, Belluzzi, \& Grossman, 1970) have been reported. One may argue perhaps that the differences in activity results are due to the different types of devices used. To be sure, this is one possible explanation, since correlations between activity and the type of apparatus used "are not significantly different from zero or are at times positive and at other times negative"' (Ferguson, 1976, p. 116). However, body weight and level of deprivation have been poorly controlled in these studies. Since these factors influence barpressing tasks, it is not unreasonable to assume that they may influence spontaneous activity in an analogous way.

It is well known that rats and other animals increase their locomotor activity when deprived of food (Baumeister, Hawkins, \& Cromwell, 1964). But again, equivocal results have been obtained with VMH-lesioned rats. Kennedy (1964) and Teitelbaum (1957) report that VMH animals fail to appreciably increase their activity during a fast, whereas Storlien and Albert (1972) have reported that VMH animals increase their activity level with food deprivation. Therefore, the present study was designed to examine the effects of body weight (nonobese vs. obese) and deprivation level on the activity of VMH-lesioned rats in a factorial design. Both photocell activity and open-field activity measures were chosen, as these have been shown to provide differential information about spontaneous activity (Archer, 1973; Krsiak, Steinberg, \& Stolerman, 1968; Walsh \& Cummins, 1976).

\section{METHOD}

\section{Animals}

The animals were 24 female Holtzman albino rats, approximately 150 days old and $230-280 \mathrm{~g}$ at the beginning of the experiment. All rats were individually caged in an animal room with a 7:00 a.m./6:00 p.m. light/dark cycle.

\footnotetext{
Apparatus

Five photocell activity cages consisted of a $17 \times 24 \times 17.5 \mathrm{~cm}$ deep Plexiglas enclosure with a wire-mesh bottom. A Heathkit (Model 6D-1021) photobeam was placed so that the light beam cut across the width of each cage. The photocell was $5 \mathrm{~cm}$ above the floor and was equidistant from either end of the cage. Each beam breakage activated an electromechanical relay, with the closure of the relay activating a counter. Each activity cage was placed in an enclosed plywood box insulated with standard utility board. Each box was illuminated by a $7-\mathrm{W}$ houselight (powered by $28 \mathrm{~V} \mathrm{dc}$ ). The open field was constructed of Royal tile Masonite $67 \times 15 \mathrm{~cm}$, and painted black with $16 \times 15 \mathrm{~cm}$ squares with white borders.
}

\section{Procedure}

Preoperatively, the rats were maintained on free access to Purina Lab Cow pellets and tap water for 14 days. For Days 4 to 14, preoperative baseline data for photocell activity and openfield measurements were obtained. In addition, the last 5 days of this period was used to establish free-feeding body weights (preoperative nonobese body weights). The rats were matched on the photocell activity measure and randomly assigned to either the VMH group $(N=14)$ or the sham control group $(N=10)$. Surgery was then performed. Two rats from the VMH group died during surgery and three others failed to reach the weight gain criterion (see Results). Thus, there were a total of 9 rats in the VMH group and 10 rats in the sham control group for data analysis.

The first postoperative testing period (nonobese test phase) did not begin until 4 days after surgery in order to reduce the immediate effects of surgical trauma. The nonobese test phase consisted of photocell activity measurement for 7 days and openfield activity measurement for 4 days at each of the following deprivation levels: $20 \%, 10 \%$, and $0 \%$ deprivation from the preoperative body weights. There were a total of 21 days of testing.

The deprivation order for the animals in each group was counterbalanced. The rats were maintained at these percent weight loss levels by adjusting their daily ration of Purina Lab Chow. Four days were allocated between each deprivation level in order to reach the approximate deprivation level. Following the nonobese test phase, all rats were given free access to food and water for 56 days and the VMH animals were allowed to demonstrate obesity. The obese test phase was then begun and duplicated the nonobese test phase, except that the deprivation levels were computed from the new, obese free-feeding weights and 7 days were used to adjust body weights between each deprivation level.

Photocell activity measurement. Photocell activity was measured once during the day (12:00 p.m.-1:00 p.m.). Each test session lasted for $15 \mathrm{~min}$, with animals run in groups of five. At least one control animal was tested with each group of five rats.

Open-field test. Between the hours of 2:30 p.m. and 3:45 p.m.; each animal was placed in the center of the open-field (to minimize the effects of handling, the home cage was tilted so the animal could leave voluntarily) and allowed $5 \mathrm{~min}$ in the field. Two observers recorded the following measures: number of squares entered, number of rears, number of feces, amount of time in resting or lying position, and number of grooms. The subjects were then transported to the home cage and fed their daily ration.

Surgery. Bilateral lesions of the ventromedial hypothalamus were produced under sodium pentobarbital (Diabutal) anesthesia $(45 \mathrm{mg} / \mathrm{kg})$ by passing a $2.0-\mathrm{mA}$ anodal current between the .5 $\mathrm{mm}$ uninsulated tip of a stainless steel electrode $(.25 \mathrm{~mm}$ in diameter, insulated with Diamel) for $20 \mathrm{sec}$. The lesion circuit was completed through a cathode connection to the tail. The electrodes were stereotaxically positioned $.6 \mathrm{~mm}$ posterior to bregma, $.6 \mathrm{~mm}$ lateral to the midline, and $9.5 \mathrm{~mm}$ below the dural surface (Pellegrino \& Cushman, 1967). A sham surgical procedure (the electrode was lowered $4.7 \mathrm{~mm}$ below the dural surface and no current was passed) for the operated controls was performed. At the conclusion of the experiment, all animals were given an overdose of sodium pentobarbital and perfused through the heart with physiological saline followed by $10 \%$ Formalin solution. The brains were removed, fixed in Formalin, embedded in gelatin, and sectioned at $50 \mu$. Every fourth section was mounted on a slide and stained with cresyl violet.

\section{Weight Gain Criterion}

A weight gain criterion, described by Porter et al. (1974), was used in order to determine whether or not the VMH-lesioned rats were hyperphagic. Animals in the VMH group were considered hyperphagic if their weight gain exceeded the one-tailed (upper limit) $95 \%$ confidence interval based on the weight gains of the sham control group $(\mathrm{N}=10)$. Three $\mathrm{VMH}$-lesioned rats which did not meet the weight gain criterion were discarded from the study, leaving nine rats in the VMH group. Table 1 shows the mean (and range) weights (grams) just prior to the nonobese test phase 
Table 1

Mean Body Weights (in Grams) for the Sham Control Rats $(\mathrm{N}=10)$ and the VMH Rats Which Met the Weight Gain Criterion $(N=9)$ Shown for Just Prior to the Nonobese Test Phase and the Obese Test Phase

\begin{tabular}{|c|c|c|c|c|c|c|}
\hline \multirow[b]{2}{*}{ Group } & \multicolumn{2}{|c|}{ Pre-Nonobese Test Phase } & \multicolumn{2}{|c|}{ Pre-Obese Test Phase } & \multicolumn{2}{|c|}{ Weight Gain } \\
\hline & Mean & Range & Mean & Range & Mean & Range \\
\hline $\begin{array}{l}\text { VMH } \\
\text { Sham Control }\end{array}$ & $\begin{array}{l}255.8 \\
256.5\end{array}$ & $\begin{array}{l}230-280 \\
234-279\end{array}$ & $\begin{array}{l}667.7 \\
343.7\end{array}$ & $\begin{array}{l}560-875 \\
320-360\end{array}$ & 411.9 & $\begin{array}{r}317-596 \\
71-115\end{array}$ \\
\hline
\end{tabular}

and the obese test phase for each group. The mean (and range) weight gain during the 56-day free-feeding period is also reported for each group.

\begin{abstract}
Histology
All VMH-lesioned rats suffered extensive bilateral damage to the VMH. The term VMH is used to refer to the baso-medial portion of the hypothalamus and is not limited to the VM nuclei; it also includes the surrounding tissue. In light of the recent controversy surrounding the exact anatomical location which is responsible for hypothalamic hyperphagia (Gold, 1973), the final criteria for an acceptable lesion was that it be bilateral and that it produce a significant weight gain. The large lesions in these animals destroyed the VM nuclei and portions of the anterior, dorsal medial, arcuate, paraventricular, and posterior hypothalamic nuclei. The three VMH-lesioned rats which did not meet the weight-gain criterion had either very small lesions or only unilateral damage.
\end{abstract}

\section{RESULTS}

\section{Behavioral Data}

The interobserver reliability coefficients for the five open-field behaviors for the two independent observers were: squares entered $r=.94(p<.01)$, time spent resting $\mathrm{r}=.89(\mathrm{p}<.01)$, rears $\mathrm{r}=$ $.95(\mathrm{p}<.01)$, feces $r=1.0(\mathrm{p}<.01)$, and grooms $r=$ $.92(\mathrm{p}<.01)$. Mean number of responses for the six activity measures at each deprivation level under each body-weight condition was obtained for the purpose of data analyses.

A $2 \times 2 \times 3$ analysis of variance was then performed each of the activity measures. Where appropriate, Duncan's multiple-range tests were used for post hoc comparisons (significance levels < .05).

Photocell activity. Figure 1 presents the mean number of photocell activity counts for each group as a function of body-weight condition and level of deprivation. There was a significant body-weight effect $(F=7.00, \mathrm{df}=1 / 16, \mathrm{p}<.025)$ and deprivation level effect $(F=15.95$, df $=2 / 32, p<.001)$. In addition, the Group by Body Weight interaction $(\mathrm{F}=11.12$, df $=1 / 16, \mathrm{p}<.005)$ and Group by Deprivation interaction $(F=3.40$, df $=2 / 32, p<$ $.05)$ were significant. Post hoc comparisons revealed that there was a significant decrease in photocell activity for the VMH rats from the nonobese test phase to the obese test phase at the $0 \%$ and $10 \%$ deprivation levels. During the nonobese test phase, the VMH rats showed significantly greater activity than the sham control rats at the $10 \%$ deprivation level. During the obese test phase, however, the VMH rats showed a significant decrease in activity at the $10 \%$ and $20 \%$ deprivation levels as compared to the sham control rats. Within-group comparisons of photocell activity at the $0 \%$ and $20 \%$ deprivation levels revealed significant increases for the sham control rats during the nonobese and obese test phase, but only during the obese test phase for the VMH rats.

Squares entered. Figure 2 shows the mean number of responses for the five open-field measures. There was a significant body-weight effect $(F=70.58$, df $=1 / 16, \mathrm{p}<.001)$ and deprivation level effect $(F=43.99$, df $=2 / 32, p<.001)$. The Group by Body Weight interaction $(F=22.01$, df $=1 / 16$, $\mathrm{p}<.001$ ) was also significant. For the VMH rats,

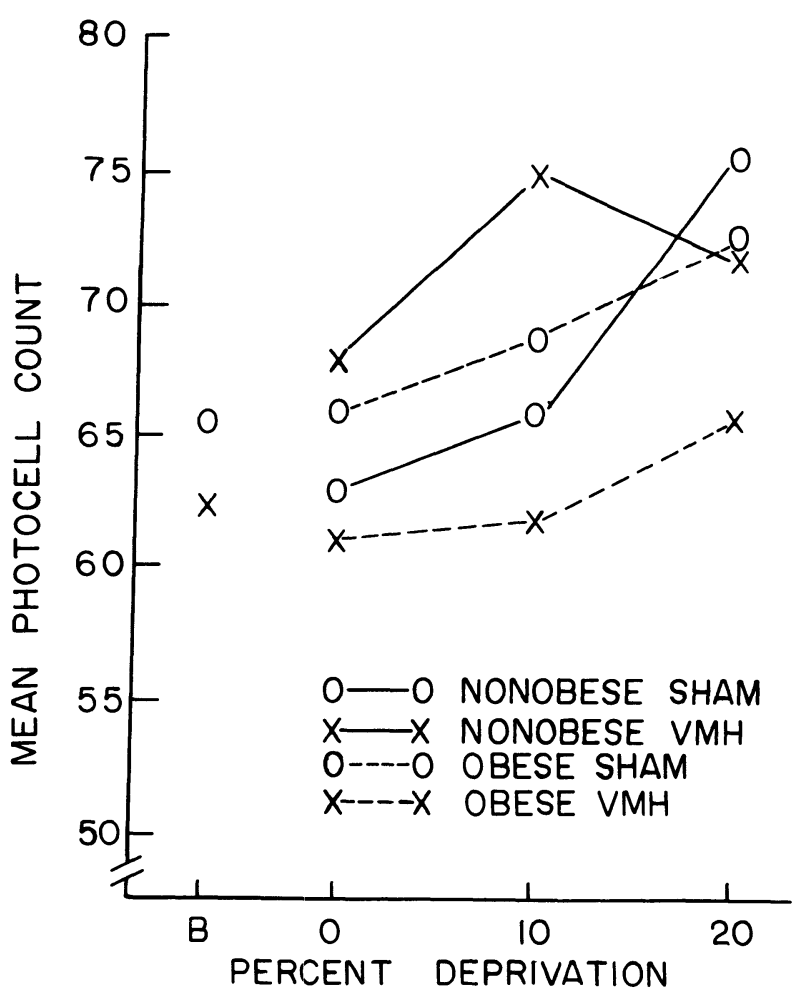

Figure 1. Mean number of photocell activity counts for each group as a function of body weight and level of deprivation. The B indicates mean activity measures taken preoperatively to establish baseline data. 

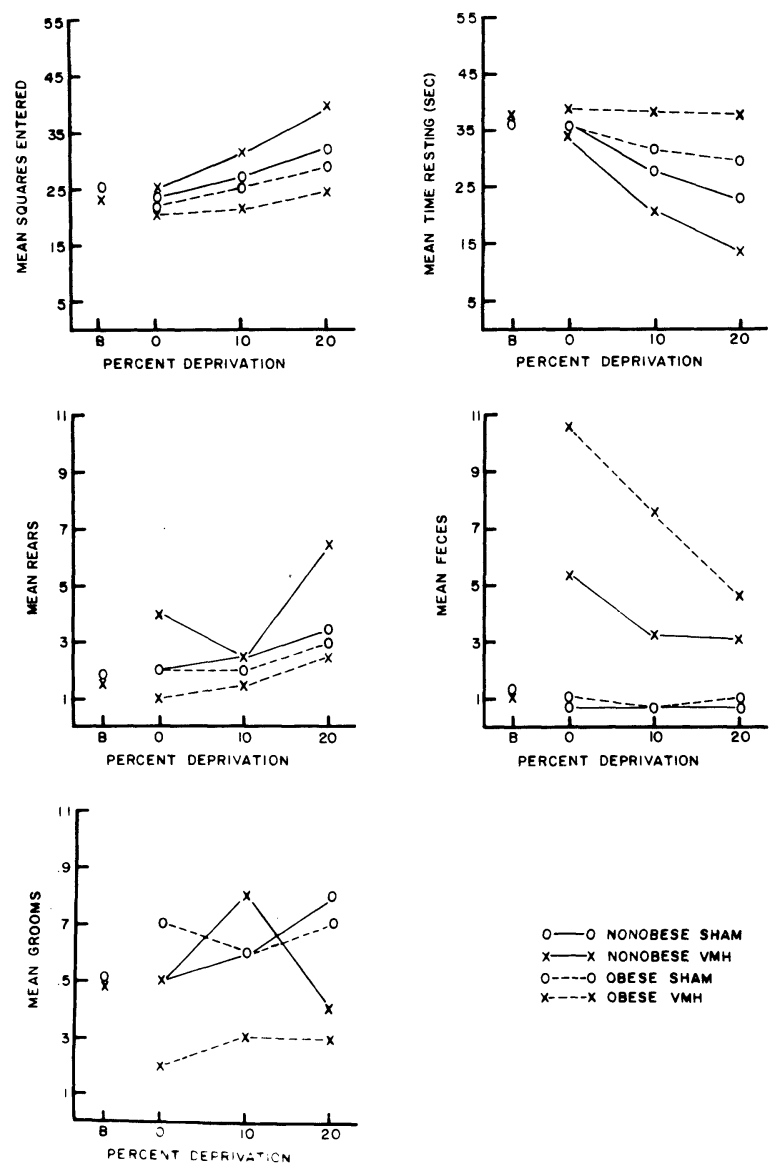

O-O NONOBESE SHAM $X$ - $\mathrm{X}$ NONOBESE VMH O-- - O OBESE SHAM

Figure 2. Mean number of responses for the five open-field measures for each group as a function of body weight and level of deprivation. The $B$ indicates mean activity measures taken preoperatively to establish baseline data.

there was a significant decrease in the number of squares entered from the nonobese test phase to the obese test phase at all three deprivation levels. The VMH rats showed a significant increase over sham control rats in squares entered at $10 \%$ and $20 \%$ deprivation during the Nonobese test phase, but showed a significant decrease in squares entered at the $20 \%$ deprivation level during the obese test phase. Within-group comparisons of $0 \%$ vs. $20 \%$ deprivation levels revealed a significant increase in number of squares entered for $\mathrm{VMH}$ and sham control rats during both phases of testing.

Time spent resting. There was a significant body weight effect $(F=16.56, \mathrm{df}=1 / 16, \mathrm{p}<.001)$ and deprivation level effect $(F=21.52$, $\mathrm{df}=$ $2 / 32, p<.001)$. The Group by Body Weight interaction $(F=6.90$, df $=1 / 16, \mathrm{p}<.025)$, Body Weight by Deprivation Level interaction $(F=17.48$, df $=2 / 32, p<.001$ ), and Group by Body Weight by Deprivation level interaction $(F=5.23$, df $=2 / 32$, $\mathrm{p}<.025$ ) were significant. There were no significant differences at the $0 \%$ deprivation level. The VMH rats spent significantly more time resting during the obese test phase than during the nonobese test phase at the $10 \%$ and $20 \%$ deprivation levels. During the nonobese test phase, the VMH rats spent significantly less time resting than the sham control rats, but spent significantly more time resting during the obese test phase at both $10 \%$ and $20 \%$ deprivation. The VMH group during the nonobese test phase and the sham control rats during both test phases showed a significant decrease in time spent resting from $0 \%$ deprivation to $20 \%$ deprivation.

Rears. For rears, the body weight $(F=27.24$, df $=1 / 16, p<.001)$ and deprivation level effects $(F=18.25, \mathrm{df}=2 / 32, \mathrm{p}<.001)$ were significant. In addition, the Group by Body Weight interaction $(F=16.48, \mathrm{df}=1 / 16, \mathrm{p}<.001)$, Body Weight by Deprivation Level interaction $(F=4.57$, $\mathrm{df}=$ $2 / 32$, p $<.025$ ), and Group by Body Weight by Deprivation Level interaction $(F=5.23, \mathrm{df}=$ $2 / 32, p<.01$ ) were significant. At the $0 \%$ and $20 \%$ deprivation levels, the $\mathrm{VMH}$ rats had a significant decrease in the number of rears during the obese test phase, as compared to the nonobese test phase. During the nonobese test phase, the VMH rats exhibited significantly more rears than the sham control rats at both $0 \%$ and $20 \%$ deprivation. During the obese test phase, the VMH rats had significantly fewer rears than the sham control rats only at the $0 \%$ deprivation level. The VMH rats at both nonobese and obese weight levels showed a significant increase in rears from $0 \%$ to $20 \%$ deprivation, but the sham control rats showed an increase only during the nonobese test phase.

Feces. The group effect $(F=96.08, \mathrm{df}=1 / 16$, $\mathrm{p}<.001)$, the body weight effect $(\mathrm{F}=9.62, \mathrm{df}=$ $1 / 16, \mathrm{p}<.01$ ), and the deprivation level effect $(F=20.88, \mathrm{df}=2 / 32, \mathrm{p}<.001)$ were significant. In addition, the Group by Body Weight interaction $(F=9.25, \mathrm{df}=1 / 16, \mathrm{p}<.01)$, the Group by Deprivation Level interaction $(\mathrm{F}=16.55$, $\mathrm{df}=$ $2 / 32, p<.001$ ), the Body Weight by Deprivation Level interaction $(F=6.14$, df $=2 / 32, p<.01)$, and the Group by Body Weight by Deprivation Level interaction $(F=4.29$, df $=2 / 32, \mathrm{p}<.025)$ were significant. The $\mathrm{VMH}$ rats had a significantly greater number of feces at obese weights than at nonobese weights at all three deprivation levels. Also, the VMH rats had a significantly greater number of feces than the sham control rats at all deprivation levels during both test phases. Withingroup comparisons revealed a significant decrease in feces count from $0 \%$ to $20 \%$ deprivation for the VMH rats during the nonobese and the obese test phases, but no significant change for the sham control rats.

Grooms. There were no significant differences between groups, between body weight levels, or across deprivation levels.

\section{Summary}

The present results indicate that the effects of 
VMH lesions on activity vary as a function of body weight of the animal and the level of food deprivation at the time of testing. Within-group comparisons of the VMH rats clearly demonstrated that the increased body weight (i.e., obese weight) decreased activity as measured by photocell and open-field activity (squares entered, time spent resting, and rears). Comparisons between nonobese VMH rats and sham control rats showed an increase level of activity (photocell, squares entered, time spent resting, and rears) but only under food-deprivation conditions $(10 \%$ and/or $20 \%)$. The only exception was at $0 \%$ deprivation for rears where the VMH rats showed a greater number of rears than the sham control rats. Decreased levels of activity (photocell, squares entered, and time spent resting) were shown by the obese VMH rats when compared to sham control rats, but once again, only during deprived testing conditions. The obese VMH rats showed a significant decrease in rears at $0 \%$ deprivation only.

The VMH rats showed a greater feces count than did sham control rats, both at nonobese and obese weights and under deprived and nondeprived conditions. Finally, there were no significant differences between groups in amount of grooming.

\section{DISCUSSION}

The present study has shown that the VMH-lesioned rat's body weight (nonobese vs. obese) and level of deprivation affect spontaneous activity, as measured by photocell and open-field activity (squares entered, time spent resting, and rears) in a way analogous to their effect on bar pressing performance (e.g., Porter \& Allen, 1977; Sclafani, 1976; Singh, 1970). The results of this study found that nonobese VMHlesioned rats display an equivalent amount of activity (except for rears) at nondeprived $(0 \%)$ testing conditions and an increased level of activity under fooddeprivation conditions $(10 \%$ and/or $20 \%)$ when compared to sham control animals. The only decrease in activity in the present study was displayed by the obese VMH-lesioned rats when they were tested under deprived testing conditions.

The VMH-lesioned rats showed a greater feces count that did sham control rats at both nonobese and obese weights and under deprived and nondeprived conditions. Since defecation in the open field has been interpreted as the prime index of emotionality (Walsh \& Cummins, 1976), the increase feces count of VMH rats, as compared to controls, indicates a change in emotionality (Grossman, 1966) and that the direction of this change is toward hyperemotionality (Singh, 1969). Singh (1969) also has stated that the hyperemotionality of these animals should show no attenuation. Results of the present study found that feces count showed a significant decrease as percent deprivation level was increased. One might be tempted to conclude that this result is contrary to Singh's hypothesis. However, Brooks, Marine, and Lambert (1946) have found that the food-feces ratio does not change with VMH animals and, therefore, the present result of decreased feces count may be due to less available food as the deprivation level was increased. The same analysis may be applied to the increased feces count of VMHlesioned animals at the obese level when compared to the nonobese level. One might consider this a further increase in emotionality, but it is more plausible to assume that the increase in feces count from the nonobese to the obese weight level simply reflects the increased body weights and food intakes of these animals at the time of testing. However, the difference in feces count at the nonobese stage between VMH and sham animals cannot be attributed to different levels of food consumption, since VMH-lesioned and sham control animals had equivalent body weights and food intakes. In sum, the results of the feces data support the notion of a change in emotionality or affective mechanisms (Grossman, 1966) and that the direction of this change is toward hyperemotionality (Singh, 1969).

Storlien and Albert (1972) investigated photocell activity of nonobese VMH-lesioned rats under deprived (19-h) and nondeprived testing conditions, and found that the VMH-lesioned rats showed less activity than control rats, with food deprivation increasing overall activity for both groups. The results of this study may seem contradictory to the present study, since no difference was obtained between the nonobese VMH group and the sham control group at the $0 \%$ deprivation level. However, a close examination of procedural differences may account for these discrepancies. In Storlien and Albert's study, activity measurement did not begin until after 7 postoperative days of ad-lib food and water. The animals gained an average of $70 \mathrm{~g}$ over the 7-day period and were considered to be in the dynamic phase of the VMH syndrome. The present study did not allow ad-lib feeding until after the nonobese test phase had ended. It is quite possible that the increased weight of the VMH animals in Storliern and Albert's study produced the decreased activity.

Open-field activity has also been investigated with VMH-lesioned rats. Green (1967) found that VMHlesioned rats traversed more squares than did controls in an open-field apparatus. It is difficult to compare Green's (1967) experiment with the present study, since body weights and feeding conditions of Green's animals were not reported. Grossman (1972) tested VMH-lesioned rats 10 weeks postoperatively (obese rats) and found that they were significantly more active, as measured by squares entered, than control rats in the open field. Grossman's (1972) VMH- 
lesioned animals may be compared to the present study's VMH-lesioned animals under the obese test phase at $0 \%$ deprivation. Since no significant difference was found between the obese VMH animals and sham controls in the present study at $0 \%$ deprivation, what could account for this discrepancy? A close look at Grossman's study reveals a series of experiments in which the VMH-lesioned animals were subjected to electric shock in order to study fighting and avoidance behavior. This may be a confounding variable that has influenced Grossman's results in the open-field test. Sclafani, Belluzzi, and Grossman, (1970) tested VMH-lesioned rats in an open field after 8 days of ad-lib feeding conditions. The activity measure (squares entered) taken during Sclafani's latency to feed test revealed no significant differences between nondeprived controls, 24- or 48-h-deprived controls, or VMH-lesioned rats. Sclafani concluded that hyperphagic rats show normal levels of activity at ad-lib nonobese hyperphagic testing conditions (as measured by squares entered). The results of Sclafani et al's (1970) data are in agreement with the present study. None of the above studies measured open-field activity of $\mathrm{VMH}-$ lesioned animals under deprived testing conditions. Thus, discrepancies between earlier research and the present study concerning the VMH-lesioned animal's activity level are probably due to procedural differences and/or the question of control over the VMH animal's body weight and level of deprivation at the time of testing. Nevertheless, what possible explanation can be offered for the nonobese VMH rats' showing an increased level of activity, but only under food-deprivation conditions? Also, obese VMH-lesioned rats show decreased levels of activity as compared to sham control rats, but once again, only during deprived testing conditions.

Recently, Mabry and Campbell (1975) and Sclafani and Rendel (1978) have offered a possible explanation of food-deprivation-induced activity in VMH rats. Mabry and Campbell (1975) found that rats with lesions of either the VMH (nonobese body weights) or the amygdala did not become hyperactive when deprived of food. However, rats with knife cuts through the lateral border of the VMH displayed an increase in food-deprivation-induced activity. They concluded that these findings supported the notion of an amygdala-hypothalamic arousal system. Since Mabry and Campbell (1975) found that food deprivation did not increase the nonobese VMHlesioned rats' activity level, and the present study did demonstrate such an effect, what could account for the difference in results? This discrepancy may be due to a procedural difference, since Mabry and Campbell (1975) allowed 14 days of ad-lib food after surgery, whereas, in the present study, food intake was restricted immediately after surgery. Sclafani and Rendel (1978) have examined terminal fooddeprivation-induced activity of rats made obese with parasagittal knife cuts through the VMH. They report that "the obese VMH-cut rats initially displayed little increase in running activity but as their weight fell to below normal levels they showed a terminal burst of activity similar to that seen in shamoperated controls"' (p. 244). Therefore, they concluded that it is the obesity, not the hypothalamic knife cut itself, that inhibits deprivation-induced activity in the VMH-cut animal. In agreement with Sclafani and Rendel's (1978) data, the present study found decreased levels of activity by the obese VMHlesioned rats when compared to sham control rats. However, this deficit in the present study was observed only during deprived testing conditions. In addition, the obese VMH-lesioned animals in the present study displayed a significant increase in activity when statistical, within-group comparisons of $0 \%$ vs. $20 \%$ deprivation levels were performed. Since the obesity of the VMH-damaged animal seems to inhibit activity, how might one interpret such an effect? Experiments by Collier (1970) and Treichler and Hall (1962) suggest that when a normal rat is subjected to at least a $10 \%$ to $15 \%$ body weight loss, it begins to show an increase in activity. Thus, these results and the reports by Mabry and Campbell (1975) and Sclafani and Rendel (1978) would suggest that some signal correlated with decreasing energy reserves stimulates locomotor activity after the reserves have fallen to some fixed critical level; although the subject of several experiments, the postulated depletion signal remains unidentified (cf. Sclafani \& Rendel, 1978). However, the data from the present experiment suggests that if body weight is stabilized by controlling available food intake, then the "criticial level" changes (increases) to a higher level in relation to the obese VMH-lesioned animal's increased weight. That is, while the obese VMH-lesioned animals' activity level was lower (under deprivation) than that of the sham controls, they (obese VMH-lesioned) significantly increased their activity as percent deprivation was increased, suggesting that the deprivation-induced increased activity was the result of the weight loss and the accompanying stimulation of some signal or signals.

Finally, the activity results in the present study seem to closely parallel the recent findings (Marks \& Remley, 1972; Porter et al., 1974; Wampler, 1973) that at nonobese weight levels the performance of VMH-lesioned rats on barpressing tasks is similar to or higher than that of normal rats, but at obese weight levels they typically exhibit a performance decrement. Taken together, these findings suggest that possibly the same signal or signals of energy reserves may be important for both barpressing tasks and spontaneous activity. 


\section{REFERENCES}

Archer, J. Tests for emotionality in rats and mice: A review. Animal Behaviour, 1973, 21, 205-235.

Baumeister, A., Hawkins, W. F., \& Cromwell, R. L. Need states and activity levels. Psychological Bulletin, 1964, 61, 438458.

BEATTY, W. W. Influence of type of reinforcement on operant responding by rats with ventromedial lesions. Physiology \& Behavior, 1973, 10, 841-846.

Brobeck, J. R., Tepperman, J., \& Long, C. N. H. Experimental hypothalamic hyperphagia in the albino rat. Yale Journal of Biology and Medicine, 1943, 15, 831-853.

Brooks, C. M. The relative importance of changes in activity in the development of experimentally produced obesity in the rat. American Journal of Physiology, 1946, 147, 708-716.

Brooks, C. M., Marine, D. N., \& Lambert, E. F. A study of the food-feces ratios and of the oxygen consumption of albino rats during various phases of experimentally produced obesity. American Journal of Physiology, 1946, 147, 717-726.

Collie r, G. H. Work: A weak reinforcer. Transactions of the New York Academy of Science, 1970, 32, 557-576.

FALK, J. L. Comments on Dr. Teitelbaum's paper. In M. R. Jones (Ed.), Nebraska symposium on motivation. Lincoln: University of Nebraska Press, 1961.

Ferguson, E. D. Motivation: An experimental approach, New York: Holt, Rinehart and Winston, 1976.

Gold, R. M. Hypothalamic obesity: The myth of the ventromedial nucleus. Science, 1973, 182, 488-490.

Green, P. C. Effects of early vs. late lesions in cognitiveeffective behavior in rats: VMH. Psychonomic Science, 1967, 7, 11-12.

Grossman, S. P. The VMH: A center for affective reactions, satiety, or both? Physiology \& Behavior, 1966, 1, 1-10.

Grossman, S. P. Aggression, avoidance, and reaction to novel environments in female rats with ventromedial hypothalamic lesions. Journal of Comparative and Physiological Psychology, 1972, 78, 274-283.

Hamilton, C. L. Interactions of food intake and temperature regulation in the rat. Journal of Comparative and Physiological Psychology, 1963, 56, 476-488.

Hetherington, A. W., \& Ranson, S. W. Hypothalamic lesions and adiposity in the rat. Anatomical Records, 1940, 78, 149-172.

Hetherington, A. W., \& Ranson, S. W. The spontaneous activity and food intake of rats with hypothalamic lesions. American Journal of Physiology, 1942, 136, 609-617.

KENNEDY, G. C. Hypothalamic control of the endocrine and behavioral changes associated with oestrus in the rat. Journal of Physiology, 1964, 172, 383-392.

Kent, M. A., \& Peters, R. H. Effects of ventromedial hypothalamic lesions on hunger-motivated behavior in rats. Journal of Comparative and Physiological Psychology, 1973, 83, 92-97.

King, B. M., \& Gaston, M. G. The effects of pretraining on the bar-pressing performance of VMH-lesioned rats. Physiology \& Behavior, 1973, 11, 161-166.

Krsiak, M., Steinberg, H., \& Stolerman, I. P. Discrepancies in results obtained with activity cages and by observation. British Journal of Pharmacology, 1968, 34, 684-685.

MabRY, P. D., \& CAMPBEll, B. A. Food deprivation-induced behavior arousal: Mediation by hypothalamus and amygdala. Journal of Comparative and Physiological Psychology, 1975, 89, 19-38.

Marks, H. E., \& Remley, N. R. The effects of type of lesion and percentage body weight loss on measures of motivated behavior in rats with hypothalamic lesions. Behavioral Biology, 1972, 7, 95-111.
Miller, N. E., Bailey, C. J., \& Stevenson, J. A. Decreased "hunger" but increased food intake resulting from hypothalamic lesions. Science, 1950, 112, 256-259.

Panksepp, J., \& Dickinson, A. On the motivational deficits after medial hypothalamic lesions. Physiology \& Behavior, 1972, 9, 609-614.

Pellegrino, L. J., \& Cushman, A. J. A stereotaxic atlas of the rat brain. New York: Appleton-Century-Crofts, 1967.

Porter, J. H., \& Allen, J. D. Food-motivated performance as a function of weight loss in hypothalamic hyperphagic rats. Psychonomic Science, 1972, 28, 285-288.

Porter, J. H., \& Allen, J. D. Food-motivation performance in rats with ventromedial hypothalamic lesions: Effects of body weight, deprivation, and preoperative training. Behavioral Biology, 1977, 19, 238-254.

Porter, J. H., Allen, J. D., \& Arazie, R. Reinforcement frequency and body weight as determinants of motivated performance in hypothalamic hyperphagic rats. Physiology \& Behavior, 1974, 13, 627-632.

Sclafani, A. Neural pathways involved in the ventromedial hypothalamus lesion syndrome in the rat. Journal of Comparative and Physiological Psychology, 1971, 77, 70-96.

Sclafani, A. Appetite and hunger in experimental obesity syndrome. In D. Novin, W. Wyrwicka, \& G. Bray (Eds.), Hunger: Basic mechanisms and clinical implications. New York: Raven Press, 1976.

Sclafani, A., Belluzzi, J. D., \& Grossman, S. P. Effects of lesions in the hypothalamus and amygdala on feeding behavior in the rat. Journal of Comparative and Physiological Psychology, 1970, 72, 394-403.

Sclafani, A., \& Kluge, L. Food motivation and body weight levels in hypothalamic hyperphagic rats: A dual lipostat model of hunger and appetite. Journal of Comparative and Physiological Psychology, 1974, 86, 28-46.

Sclafani, A., \& Rendel, A. Food deprivation-induced activity in normal and hypothalamic obese rats. Behavioral biology, 1978, 22, 244-255.

SinGH, D. Comparison of hyperemotionality caused by lesions in the septal and ventromedial areas in the rat. Psychonomic Science, 1969, 16, 3-4.

Singh, D. Sex differences in obesity and food-directed activity in normal and hyperphagic rats. Psychonomic Science, 1970, 21, 306-308.

SingH, D. Effects of preoperative training on food-motivated behavior of hypothalamic hyperphagic rats. Journal of Comparative and Physiological Psychology, 1973, 84, 47-52.

Storlien, L. H., \& Albert, D. J. The effect of VMH lesions, lateral cuts and anterior cuts on food intake, activity level, food motivation, and reactivity to taste. Physiology \& Behavior, 1972, 9, 191-197.

Teitelbaum, P. Random and food-directed activity in hyperphagic and normal rats. Journal of Comparative and Physiological Psychology, 1957, 50, 486-490.

Treichle R, F. R., \& Hall, J. F. The relationship between deprivation weight loss and several measures of activity. Journal of Comparative and Physiological Psychology, 1962, 55, 346-349.

Walsh, R. N., \& Cummins, R. A. The open-field test: A critical review. Psychological Bulletin, 1976, 83, 482-504.

WAMPLER, R. S. Increased motivation in rats with ventromedial hypothalamic lesions. Journal of Comparative and Physiological Psychology, 1973, 84, 275-285.

(Received for publication September 25, 1978; revision accepted November 3,1978 .) 